\title{
HST/WFC3 OBSERVATIONS OF AN OFF-NUCLEAR SUPERBUBBLE IN ARP 220
}

\author{
Kelly E. Lockhart ${ }^{1}$, Lisa J. Kewley ${ }^{2}$, Jessica R. Lu ${ }^{1}$, Mark G. Allen ${ }^{3}$, David Rupke ${ }^{4}$, Daniela Calzetti ${ }^{5}$, \\ Richard I. Davies $^{6}$, Michael A. Dopita ${ }^{2}$, Hauke Engel ${ }^{6}$, Timothy M. Heckman ${ }^{7}$, \\ Claus Leitherer $^{8}$, ANd David B. Sanders ${ }^{1}$ \\ ${ }^{1}$ Institute for Astronomy, 2680 Woodlawn Drive, Honolulu, HI 96822, USA \\ ${ }^{2}$ Research School of Astronomy and Astrophysics, Australian National University, Cotter Road, Weston Creek ACT 2611, Australia \\ ${ }^{3}$ Observatoire de Strasbourg, UMR 7550, Strasbourg, F-67000, France \\ ${ }^{4}$ Department of Physics, Rhodes College, Memphis, TN 38112, USA \\ ${ }^{5}$ Department of Astronomy, University of Massachusetts, Amherst, MA 01003, USA \\ ${ }^{6}$ Max-Planck-Institut für Extraterrestrische Physik, Postfach 1312, D-85741 Garching, Germany \\ ${ }^{7}$ Center for Astrophysical Sciences, Department of Physics and Astronomy, Johns Hopkins University, Baltimore, MD 21218, USA \\ ${ }^{8}$ Space Telescope Science Institute, 3700 San Martin Drive, Baltimore, MD 21218, USA \\ Received 2015 March 7; accepted 2015 June 8; published 2015 September 9
}

\begin{abstract}
We present a high spatial resolution optical and infrared study of the circumnuclear region in Arp 220, a late-stage galaxy merger. Narrowband imaging using Hubble Space Telescope/WFC3 has resolved the previously observed peak in $\mathrm{H} \alpha+\left[\mathrm{N}_{\mathrm{II}}\right]$ emission into a bubble-shaped feature. This feature measures 1". 6 in diameter, or $600 \mathrm{pc}$, and is only $1^{\prime \prime}$ northwest of the western nucleus. The bubble is aligned with the western nucleus and the large-scale outflow axis seen in X-rays. We explore several possibilities for the bubble origin, including a jet or outflow from a hidden active galactic nucleus (AGN), outflows from high levels of star formation within the few hundred pc nuclear gas disk, or an ultraluminous X-ray source. An obscured AGN or high levels of star formation within the inner $\sim 100 \mathrm{pc}$ of the nuclei are favored based on the alignment of the bubble and energetics arguments.
\end{abstract}

Key words: galaxies: evolution - galaxies: individual (Arp 220) - galaxies: ISM

\section{INTRODUCTION}

Galaxy mergers are integral to understanding galaxy evolution. Simulations suggest that major mergers cause large-scale tidal gas inflows, which may fuel nuclear starbursts and active galactic nucleus (AGN) activity (Barnes \& Hernquist 1996; Mihos \& Hernquist 1996). In later stages, mergers may host young, obscured AGNs, which, simulations predict, are gradually uncovered as AGN feedback and high levels of circumnuclear star formation expel the surrounding gas (Barnes \& Hernquist 1996; Mihos \& Hernquist 1996; Di Matteo et al. 2005; Hopkins et al. 2005; Hopkins \& Hernquist 2006). This nuclear activity can, in turn, fuel large-scale galactic outflows (Heckman 2003; Veilleux et al. 2005), which have been observed in many merging systems (Heckman et al. 1990, 2000; Rupke et al. 2002, 2005a, 2005b, 2005c; Martin 2005, 2006; Rich et al. 2010; Rupke \& Veilleux 2011, 2013b).

Large-scale outflows in the outer parts of galaxies have been extensively studied. However, the advent of high spatial resolution observations has prompted more recent studies to probe the launch sites, propagation, and escape of these outflows (Rupke \& Veilleux 2013a; Emonts et al. 2014; Sakamoto et al. 2014). When observed with sufficient spatial resolution, an outflow still within a galaxy often has complex superbubble or filamentary structure (Veilleux et al. 1994; Forbes et al. 2000; Cecil et al. 2001; Kenney \& Yale 2002), created as warmer, high-velocity material ejected from a galaxy's nuclear region interacts with the cooler interstellar medium (ISM). However, these well-shaped structures have thus far not been observed in the turbulent, clumpy ISM of a late-stage merger. In addition, while both the timescale and impact of AGN feedback into the host system have been calculated theoretically (Hopkins et al. 2005; Hopkins \& Elvis 2010), both are still observationally uncertain.
Investigating an individual late-stage merging system that exhibits both an outflow and a possible obscured AGN may provide clues to this process.

Arp 220 is the closest ultraluminous infrared galaxy (ULIRG, $L_{\mathrm{IR}}>10^{12} L_{\odot}$, Soifer et al. 1987; Sanders et al. 1988). The system has long been recognized as a late-stage merger, as evidenced by extended tidal tails visible in the optical (Arp 1966; Surace et al. 2000), infrared (Joseph \& Wright 1985), and in H I (Hibbard et al. 2000), as shown in Figure 1. It also hosts a large-scale outflow that extends far from the galaxy. Arp 220 has a molecular gas mass of $\sim 10^{10}$ $M_{\odot} \quad$ (Scoville et al. 1997) and a total star formation rate, calculated using the far-infrared luminosity, of $240 \pm$ $30 M_{\odot} \mathrm{yr}^{-1}$ (Farrah et al. 2003). Most of the molecular gas and star formation are concentrated within a central few $100 \mathrm{pc}$ nuclear gas disk, which also hosts a double nucleus. A possible AGN has been suggested (Paggi et al. 2013) but is still debated. At a distance of $77 \mathrm{Mpc}$, its close proximity offers a unique laboratory to investigate the interaction of its outflow with the ISM at small spatial scales, and to determine how energy propagates out of the interior of such a system. A more detailed background on the structure of Arp 220 is presented below.

Heckman et al. (1987) observed large-scale $(10 \mathrm{kpc})$ bipolar lobes extending from the galaxy via narrowband $\mathrm{H} \alpha$ imaging, which they interpret as a superwind. Further spectroscopic study revealed these lobes to be superwind-driven bipolar bubbles (Heckman et al. 1990). This superwind is also observed in soft X-ray emission (McDowell et al. 2003).

In addition to the large-scale wind, smaller-scale gas dynamics have been investigated in more recent optical IFU studies. Colina et al. (2004) found large-scale plumes and lobes, up to $8 \mathrm{kpc}$ in length and aligned with the superwind, which are consistent with high-velocity shocks expanding in a 

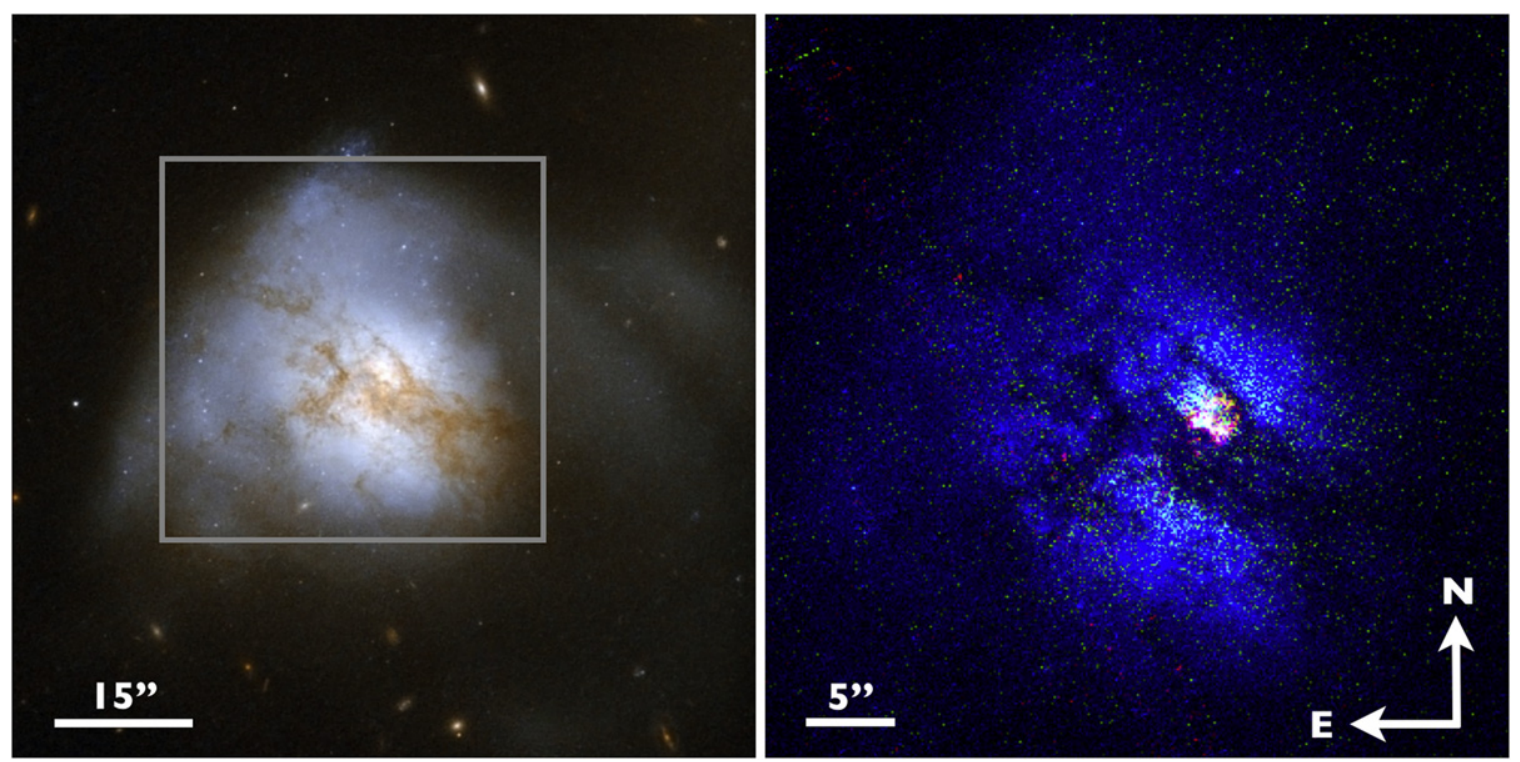

Figure 1. Left: HST ACS image of Arp 220 as first presented in Wilson et al. (2006), rotated to adjust for the $77^{\circ}$ position angle. The blue image is taken with the F435W filter and the red with the F814W filter (green in a linear combination of the red and blue filters). The gray box indicates the FOV of the zoomed right panel. Right: our new data taken with HST WFC3. Blue is the F467M blue continuum image, green is [O III], or the FQ508N image with the blue continuum subtracted, and red is $\mathrm{H} \alpha+[\mathrm{N}$ II $]$, or the F665N image with the red continuum subtracted. North is up, east is left.

neutral medium. Arribas et al. (2001) studied the inner region of the system within $2 \mathrm{kpc}$ of the nuclei and found three gas components. One component is coupled to the central molecular gas disk. The other two components are associated with a biconal outflow or superwind.

This outflow, whether star formation- or AGN-driven, almost certainly originates within the nuclear region of the galaxy. At the center of Arp 220, two dust-enshrouded nuclei, separated by 0 ".98 (368 pc), are visible in the IR (Scoville et al. 1998), sub-mm (Scoville et al. 1997; Downes \& Solomon 1998; Sakamoto et al. 1999; Scoville et al. 2014), and radio (Baan \& Haschick 1995). Sakamoto et al. (1999) resolved the large circumnuclear gas mass in a detailed CO study, and found that each nucleus is embedded in its own gas disk, each roughly $100 \mathrm{pc}$ in radius, and these disks are counterrotating with respect to each other and inclined such that their near sides are to the south. The position angle (P.A.) of each of these small disks is $52^{\circ}$ and $263^{\circ}$ for the eastern and western nuclei, respectively, while that of the larger circumnuclear disk is $25^{\circ}$. Scoville et al. (2014) calculated the ISM masses of each nuclear gas disk, finding a mass of 1.9 (4.2) $\times 10^{9} M_{\odot}$ around the eastern (western) nucleus, concentrated within a radius $69(65) \mathrm{pc}$ of the nucleus. These small gas disks are surrounded by a larger gas disk $\sim 1 \mathrm{kpc}$ in radius, which rotates around the dynamical center of the system (Sakamoto et al. 1999). Mundell et al. (2001) used observations of $\mathrm{H}_{\mathrm{I}}$ in the circumnuclear gas disks and comparison with simulations to estimate that the merger started about $700 \mathrm{Myr}$ ago, with the second, most recent starburst starting in the western nuclear gas disk more recently than 10-100 Myr ago.

Within the inner $50 \mathrm{pc}$ of each nucleus, the average molecular gas density is $\sim 10^{5} \mathrm{~cm}^{-3}$ (Scoville et al. 2014), comparable to the conditions in a dense star-forming core inside a giant molecular cloud. Indeed, Arp 220 is undergoing very high rates of star formation. Smith et al. (1998) estimate the circumnuclear star formation rate at $50-800 \mathrm{M}_{\odot} \mathrm{yr}^{-1}$ using the radio supernovae rate, while Farrah et al. (2003) use the far- infrared luminosity to estimate a global star formation rate of $240 \pm 30 M_{\odot} \mathrm{yr}^{-1}$.

Outside of the heavily extincted region near the nuclei, star formation activity was observed with Hubble Space Telescope (HST) ACS. Wilson et al. (2006) found two populations of massive star clusters: young ( $<10 \mathrm{Myr})$ massive clusters within $2.3 \mathrm{kpc}$ of the nuclei and intermediate age $(\sim 100 \mathrm{Myr})$ clusters beyond this radius.

In this paper, we investigate the connection between the nuclear energy sources and the large-scale outflow. We use HST/WFC3 with narrowband filters to create the first high spatial resolution optical emission line maps of Arp 220. We present maps of $\left[\mathrm{O}_{\mathrm{III}}\right], \mathrm{H} \beta$, and $\mathrm{H} \alpha+\left[\mathrm{N}_{\mathrm{II}}\right]$ to resolve gas motions in Arp 220. We discover a bubble feature in $\mathrm{H} \alpha+[\mathrm{N}$ II] (Section 3) and discuss its origins in Section 4.

Throughout this work, we assume a distance of $77 \mathrm{Mpc}$, which gives $1^{\prime \prime}=375 \mathrm{pc}$.

\section{OBSERVATIONS}

\subsection{HST}

The optical imaging data of Arp 220 used in this work are new observations obtained with HST Wide Field Camera 3 (WFC3-UVIS) on 2012 September 22 (GO-12552, PI: Lisa Kewley). We selected filters whose bandpasses each cover one or more of several strong optical emission lines commonly used for calculating gas-phase metallicity and shock excitation: $\mathrm{H} \beta$ (filter FQ492N); [O III] $\lambda \lambda$ 4959, 5007 (rest; FQ508N); $\mathrm{H} \alpha+$ $[\mathrm{N}$ II $] \lambda \lambda$ 6548, $6583(\mathrm{~F} 665 \mathrm{~N})$; and $\left[\mathrm{S}_{\mathrm{II}}\right] \lambda \lambda$ 6717, 6731 $(\mathrm{F} 680 \mathrm{~N})$. Blue $(\mathrm{F} 467 \mathrm{M})$ and red $(\mathrm{F} 621 \mathrm{M})$ continuum exposures were also taken to allow calculation of the line flux across the extent of the system. Figure 2 shows the filter bandpasses superimposed over the SDSS 7 spectrum of Arp 220. The filter and exposure data are summarized in Table 1 along with the continuum bandpass used with each emission line image and the $1 \sigma \mathrm{rms}$ errors (discussed further in Sections 2.1.1 and 2.1.2) With a pixel size of 0 ".0396, the field 


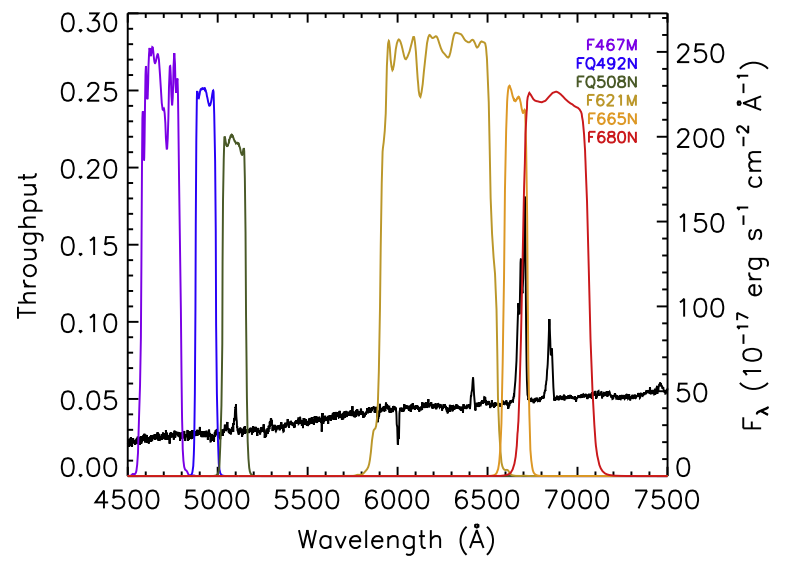

Figure 2. SDSS 7 spectrum of Arp 220 with the WFC3-UVIS filter bandpasses overlaid. The left axis shows the throughput of each WFC3 filter (colors are as shown in the legend) while the right axis shows the flux of the SDSS spectrum (in black).

of view (FOV) of WFC3-UVIS is $164^{\prime \prime} \times 176^{\prime \prime}$ as measured on the image. The FQ, or quad, filters, each occupy one-quarter of the FOV, so the FOV for these filters is approximately $82^{\prime \prime} \times 88^{\prime \prime}$.

Data reduction was performed using the STScI pipeline, which utilizes "On-the-fly Reprocessing." This processes the raw data files using the best calibration files and software available at the time of retrieval from the archive and performs basic bias and dark subtraction and flat fielding. It also flags bad pixels and performs initial cosmic ray removal. The data were all retrieved from the archive in late 2012.

The reduced dithered exposures were combined using AstroDrizzle (Avila et al. 2012). AstroDrizzle, the successor to MultiDrizzle, provides distortion corrections and combines multiple images using a technique which allows for image subsampling. Sky subtraction was performed using the mode of the image, in order to not bias the sky value by the extended nebulosity of the galaxy. Charge transfer efficiency (CTE) trails caused by inefficient electron shuffling during readout were evident on the initial combined images. Due to the high counts involved, they were especially problematic in the pixels along which cosmic ray hits were shuffled. The charge in the first smeared pixel amounted to roughly $1 \%$ of the initial high pixel value and dropped off rapidly as the charge was further shuffled along the detector. However, the CTE trail left by each cosmic ray was up to 20 pixels long for the strongest cosmic rays. These pixels were masked in AstroDrizzle, as the data were downloaded and reduced before the CTE correction software from STScI became available. All other AstroDrizzle parameters were set to the default values. The final combined images were registered to a common reference frame using the AstroDrizzle tasks tweakreg and tweakback and redrizzled onto the same pixel grid, to allow for image combination.

The FQ492N and FQ508N filters, covering $\mathrm{H} \beta$ and [O III], had a sufficient number of exposures to allow for cosmic ray removal (see Table 1). The other filters (F467M, F621M, F665N, F680N) had two dithered exposures each; the cosmic ray cleaning provided by AstroDrizzle for these was not robust, especially in the gap between the two detector chips. The final combined images for these filters were further cleaned of cosmic rays using a Laplacian edge detection algorithm, which identifies cosmic rays based on the sharpness of their edges (van Dokkum 2001). This method, which removes only the positive noise spikes, can alter the noise distribution of the image. The resulting noise distribution may be asymmetric and skewed toward negative values, which can be problematic for low signal-to-noise ratio $(\mathrm{S} / \mathrm{N})$ regions. We examined the cleaned images and found that only the F665N image is affected. Because we focus on the high $\mathrm{S} / \mathrm{N}$ regions in this filter, this does not affect our results.

The bandpass of one of the continuum images, through filter F621M, contains $\left[\mathrm{O}_{\mathrm{I}}\right] \lambda 6300 \AA$, a strong emission line in shock-excited gas. The strength of this line varies with metallicity and excitation source and thus could contaminate calculations of emission line ratios. The contribution of the flux of this line to the total flux in the continuum image was estimated using the Sloan Digital Sky Survey (SDSS) spectrum of Arp 220. The SDSS fiber covers the inner $3^{\prime \prime}$ of the galaxy, or roughly $1 \mathrm{kpc}$. The ratio of flux in the emission line to flux in the continuum contained in the bandpass was roughly $1 \%$, or less than the average error in the images. Thus, the contribution of the line to the continuum image was taken to be negligible and no correction to the continuum image was made.

The data were binned in square bins 3 pixels wide to increase the $\mathrm{S} / \mathrm{N}$. At the redshift of $0.018126 \pm 0.000023$ (de Vaucouleurs et al. 1991), the WFC3 pixel scale of 0".0396/ pixel corresponds to $15 \mathrm{pc}$. Extragalactic giant $\mathrm{H}$ if regions range from 50 to $300 \mathrm{pc}$ in diameter (Oey et al. 2003). The binned resolution elements measure $45 \mathrm{pc}$ across and thus allow full sampling of all but the smallest $\mathrm{H}_{\text {II }}$ regions while still allowing recovery of low signal.

\subsubsection{Continuum Subtraction}

One of the most sensitive calibration tasks is subtraction of the stellar continuum from the emission line images. Small changes in the factor used to scale the continuum before subtracting it from the emission line image propagate into a change in the flux of the final emission line image. This can have a large effect on the emission line flux and ratio maps.

We determined the optimal scale factor between the continuum image and the emission line image using the "skewness transition method" (Hong et al. 2014). This method uses the symmetry of the flux distribution within an aperture to identify the ideal scaling factor that neither under nor over subtracts the continuum. It accounts for spatially dependent luminosity changes, but not spatially dependent color changes. The location of the aperture used to calculate the scaling factor influences the derived value, and allows us to estimate an error on the scaling factor. The error on the scaling factor can be taken as 0.05 for each filter.

We verified the accuracy of this method via physical arguments. For a star cluster with zero extinction, and assuming Case B recombination, the ratio of $\mathrm{H} \alpha$ to $\mathrm{H} \beta$ is 2.86. However, Arp 220 has high levels of patchy extinction and a high and varying ratio of $[\mathrm{N} \mathrm{II}]$ to $\mathrm{H} \alpha$, so we would not expect this ratio to hold across most of this system. We are able to check the ratio between the two images using a star cluster with significant $\mathrm{H} \alpha+\left[\mathrm{N}_{\mathrm{II}}\right]$ and $\mathrm{H} \beta$ emission, and a measured $E(B-V)$ of 0 (Wilson et al. 2006 their cluster 62). We assumed that the ratio of $\left[\mathrm{N}\right.$ II] to $\mathrm{H} \alpha$ is roughly that for an $\mathrm{H}_{\text {II }}$ region ( $\sim 0.3$, Kewley et al. 2006a) and adjusted the scaling factor for the continuum subtraction from the $\mathrm{H} \beta$ image until we achieve a ratio equal to the Balmer decrement. With this method, we calculate a new scaling factor of $0.49 \pm 0.02$. This 
Table 1

$H S T$ WFC3-UVIS Exposures

\begin{tabular}{|c|c|c|c|c|c|c|c|}
\hline \multirow[b]{2}{*}{ Filter } & \multicolumn{2}{|c|}{ Bandpass } & \multirow[b]{2}{*}{ Line } & \multirow[b]{2}{*}{ Exposures } & \multicolumn{2}{|c|}{ Continuum } & \multirow{2}{*}{$\begin{array}{c}\text { Flux Error } \\
1 \sigma^{\mathrm{b}}\left(\mathrm{erg} \mathrm{s}^{-1} \mathrm{~cm}^{-2} \AA^{-1} \operatorname{arcsec}^{-2}\right)\end{array}$} \\
\hline & Central $\lambda(\AA)$ & Width $(\AA)$ & & & Filter & Scale $^{\mathrm{a}}$ & \\
\hline$\overline{\mathrm{FQ} 492 \mathrm{~N}}$ & 4933 & 114 & $\mathrm{H} \beta$ & $2 \times 1090 \mathrm{~s}, 6 \times 920 \mathrm{~s}$ & F467M & 0.42 & $3.9 \times 10^{-18}$ \\
\hline FQ508N & 5091 & 131 & [O III] 4959, 5007 & $2 \times 1050 \mathrm{~s}, 6 \times 920 \mathrm{~s}$ & F467M & 0.46 & $3.8 \times 10^{-18}$ \\
\hline F665N & 6656 & 131 & $\mathrm{H} \alpha+[\mathrm{N}$ II $] 6548,6583$ & $2 \times 200 \mathrm{~s}$ & F621M & 0.16 & $1.4 \times 10^{-17}$ \\
\hline F680N & 6877 & 371 & {$[\mathrm{~S} \text { II }]^{\mathrm{c}} 6717,6731$} & $2 \times 525 \mathrm{~s}$ & F621M & 0.66 & $7.7 \times 10^{-18}$ \\
\hline F467M & 4683 & 201 & Blue continuum & $2 \times 775 \mathrm{~s}$ & $\ldots$ & $\cdots$ & $\cdots$ \\
\hline F621M & 6219 & 609 & Red continuum & $2 \times 155 \mathrm{~s}$ & $\ldots$ & $\ldots$ & $\ldots$ \\
\hline
\end{tabular}

Notes.

a Scaling factor of the continuum used for continuum subtraction.

${ }^{b}$ Flux errors are estimated based on the dispersion of the sky background in each image, as measured on a section of sky away from the galaxy.

${ }^{c}$ Not used for analysis due to shallowness of image; see appendix.

scaling factor is generally consistent with that obtained through the skewness transition method $(0.42 \pm 0.05)$, and so we adopt the scaling factors from the skewness transition method. Our scaling factors are given in Table 1.

\subsubsection{Error Estimation}

Flux errors are estimated based on the standard deviation of the background in each image. The dispersion varies slightly across the field, so to obtain a representative value this was measured in a region centered within $2^{\prime \prime}$ of the detected emission line flux in each filter, but not overlapping it. We show the $1 \sigma$ errors in Table 1 . We adopt an S/N cutoff of $4 \sigma$ in all emission line images. However, the F680N image was too shallow and we were unable to recover [S II] flux above the $4 \sigma$ limit (see appendix). We thus drop this image from further analysis.

\subsection{Other Data}

\subsection{1. $\mathrm{H}_{2}$ Data}

Near-infrared (near-IR; $\mathrm{H}$ and $\mathrm{K}$ band) integral field spectroscopy (IFS) data were taken 2007 March 7 and April 18-21 with SINFONI, a NIR integral field spectrometer on the Very Large Telescope (Eisenhauer et al. 2003). The curvaturebased adaptive optics system was operated in laser guide star mode, though without tip-tilt correction as no suitable tip-tilt star was available. This gave a nearly symmetric Gaussian point spread function of 0 ". $30 \times 0$ ". 31 . The 0 ". $05 \times 0$ ". 10 plate scale was used, giving a FOV of $3^{\prime \prime} .2 \times 3^{\prime \prime}$. 2 . Further details on the observations, data reduction, and line fitting are given in Engel et al. (2011).

\subsubsection{NICMOS}

NIR imaging using HST/NICMOS was obtained from the $H S T$ archives. The F160W filter on camera NIC2 was used to image Arp 220 in the H-band on 10 January 2004 (proposal 9726, PI: R. Maiolino) for a total exposure time of $600 \mathrm{~s}$ (Cresci et al. 2007). The F222M filter (K band) on NIC2 was used to image the system on 4 April 1997 for a total exposure time of $1024 \mathrm{~s}$ (Scoville et al. 1998, proposal 7116, PI: N. Scoville). The pixel scale on the NIC2 camera is 0 ". 076 while the FOV is $19^{\prime \prime} .2$.

\subsection{Registration}

There are no stars suitable for image registration in the FOV. Instead, bright super star clusters (SSCs) from Wilson et al. (2006) were used to align the WFC3 and NICMOS datasets. Three bright, well-separated clusters were used, allowing a correction for both shift and rotation between the images. The absolute astrometric registration was set by the coordinates of the western nuclei given by Scoville et al. (1998), precessed to J2000.0.

The SINFONI data have a smaller FOV, limited to the circumnuclear region. The optical SSCs previously used for alignment were out of this FOV. Instead, the IFU data were aligned directly with the NICMOS F222M image using the nuclei and another bright infrared source.

\section{MORPHOLOGY}

\subsection{Optical Emission Line Structure}

We present emission line maps of $[\mathrm{O}$ III], $\mathrm{H} \beta$, and $\mathrm{H} \alpha+[\mathrm{N} \mathrm{II}]$ in Figure 3. Each image is $45^{\prime \prime} \times 45^{\prime \prime}$. A $4 \sigma \mathrm{S} / \mathrm{N}$ cut has been applied, and the logarithm of the emission is displayed in order to show the full range of emission. The maps have been rotated to account for a $77^{\circ}$ P.A., so that north is up and east is left. The NIR locations of the double nuclei (Scoville et al. 1998) are shown as red diamonds. Some residual cosmic rays are visible in the chip gap of the $\mathrm{H} \alpha+[\mathrm{N}$ II] (right) panel Figure 3, as detailed in Section 2.

The imaging reveals widespread $[\mathrm{O}$ III] and $\mathrm{H} \beta$ emission across the central disk, roughly $8 \mathrm{kpc}$ in diameter. Faint tidal tails are visible to the northeast. A dust lane runs from the northeast to the southwest, obscuring the double nuclei of the system, which are only visible in the X-ray, IR, and radio. Though visible across the main disk of the system, the [O III] and $\mathrm{H} \beta$ emission is concentrated near the optical center, which is roughly $1^{\prime \prime} .5$ offset to the northwest from the western nucleus. The $\mathrm{H} \alpha+\left[\mathrm{N}_{\mathrm{II}}\right]$ emission is much more tightly concentrated near the optical center. We also present emission line maps zoomed into the nuclear region in Figure 4.

The most striking feature in the emission line maps is a ringor bubble-shaped structure, seen in $\mathrm{H} \alpha+[\mathrm{N} \mathrm{II}]$ emission (Figure 4, right panel). It is unclear whether the feature is due to strong emission in $[\mathrm{N}$ II], $\mathrm{H} \alpha$, or both. Strong $\mathrm{H} \alpha$ emission indicates high rates of star formation, while high [N II] emission generally indicates the presence of shocked gas (see Kewley et al. 2006a). The ring measures 1".6 in diameter, or $600 \mathrm{pc}$ at the distance of $\operatorname{Arp} 220$, with a P.A. of $316^{\circ}$ as 

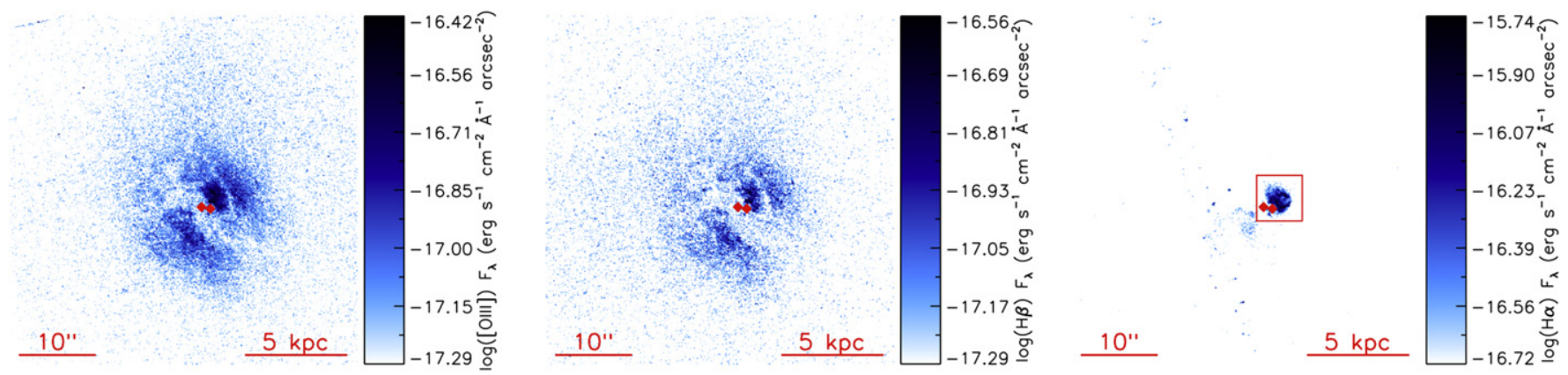

Figure 3. Maps of the $\log ([\mathrm{O}$ III $])$ (left), $\log (\mathrm{H} \beta)$ (middle), and $\log \left(\mathrm{H} \alpha+[\mathrm{N}\right.$ II $]$ ) (right) emission across the galaxy, rotated to account for a $77^{\circ}$ P.A. so that north is up and east is left. The locations of the eastern and western nuclei, as determined in the near-IR by Scoville et al. (1998) and precessed to J2000.0, are shown as red diamonds. A box showing the size of the zoomed Figure 4 is shown on the right panel. A $4 \sigma \mathrm{S} / \mathrm{N}$ cut has been applied.
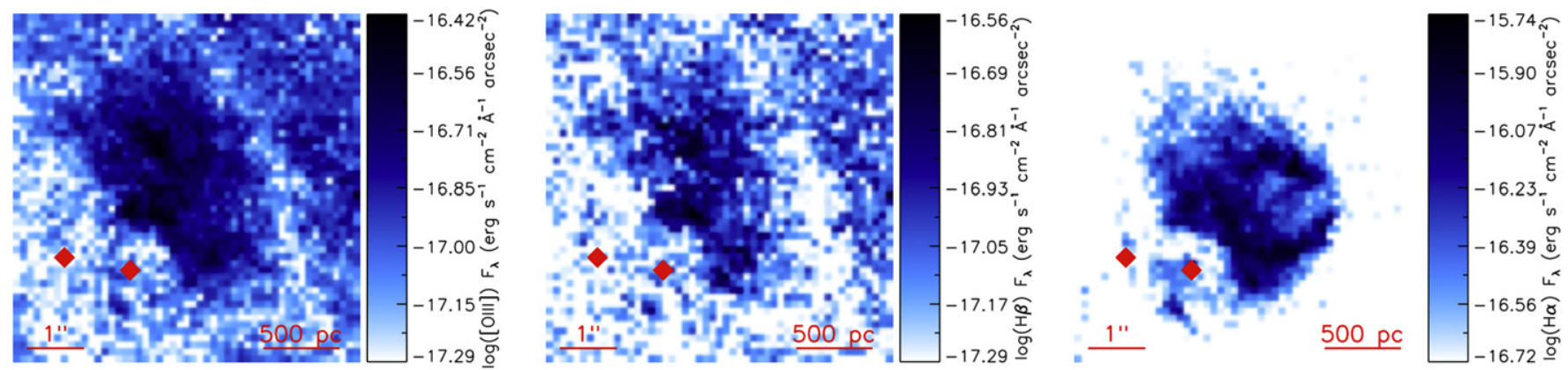

Figure 4. Map of the $\log ([\mathrm{O}$ III $])$ (left), $\log (\mathrm{H} \beta)$ (middle), and $\log \left(\mathrm{H} \alpha+[\mathrm{N}\right.$ II] $)$ (right) emission near the nuclear region, zoomed in on a central $6^{\prime \prime} \times 6^{\prime \prime}$ region. As in the full map, a $4 \sigma \mathrm{S} / \mathrm{N}$ cut has been applied, the locations of the eastern and western nuclei are shown as red diamonds, and the maps have been rotated so that north is up and east is left.
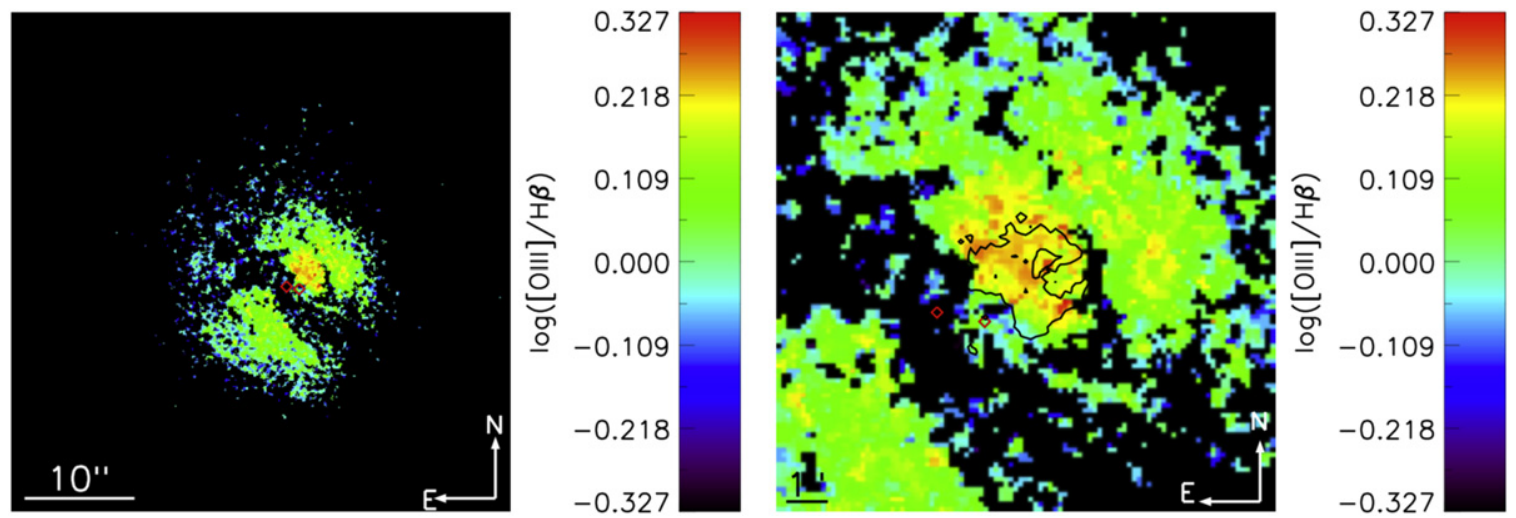

Figure 5. Median smoothed maps $(\mathrm{kernel}=3)$ of $\log ([\mathrm{O} \mathrm{mI}] / \mathrm{H} \beta)$. Red diamonds show the positions of the nuclei identified in the NIR and given in Scoville et al (1998). Left: full view of system. Right: same as left, but zoomed into the optical center of the system. In addition, contours showing the bubble feature, in $\mathrm{H} \alpha+[\mathrm{N}$ II] emission, are shown in black. North is up, east is left in both panels.

measured from the western nucleus to the bubble center. There appears to be a faint smaller ring, inside the larger one, on the side closest to the nuclei. This is the first time the $\mathrm{H} \alpha$ and [N II] peaks reported in Arribas et al. (2001, their Figure 1, approximately corresponding to their fibers $110-114$ ) have been resolved into a bubble morphology. This bubble feature and its environment are discussed further in Section 3.3.

\subsection{Emission Line Ratios}

The ratios of strong optical emission lines are commonly used as a diagnostic to determine the excitation mechanism of ionized gas (Baldwin et al. 1981; Kewley et al. 2001, 2006b; Kauffmann et al. 2003): photoionization by massive stars, excitation via shocks, or ionization from an AGN. The most widely used diagnostics require the ratio of both $[\mathrm{O}$ III] to $\mathrm{H} \beta$ and either $[\mathrm{N}$ II $],\left[\mathrm{S}_{\mathrm{II}}\right]$, or $\left[\mathrm{O}_{\mathrm{I}}\right] \lambda 6300 \AA$ to $\mathrm{H} \alpha$.

In Figure 5, we present maps of $\log \left(\left[\mathrm{O}_{\mathrm{III}}\right] / \mathrm{H} \beta\right)$, which were created from the left and center panels of Figure 3. An S/N cut of $4 \sigma$ was first applied to each individual emission line map and the resulting ratio map was smoothed using a median filter $($ kernel $=3)$ in order to emphasize larger-scale features. The right panel of Figure 5 is the same, but cropped to more closely show the region around the bubble feature. $\mathrm{H} \alpha+\left[\mathrm{N}_{\text {III }}\right]$ contours are overlaid in black on the right panel, to show the location of the bubble feature.

Some structure is visible in the smoothed maps, and is more readily apparent in the normalized histograms of $\log ([\mathrm{O}$ III $] / \mathrm{H} \beta)$ shown in Figure 6. Values for the entire system are shown in blue, while values within just the $\mathrm{H} \alpha+[\mathrm{N}$ II $]$ ring feature are 


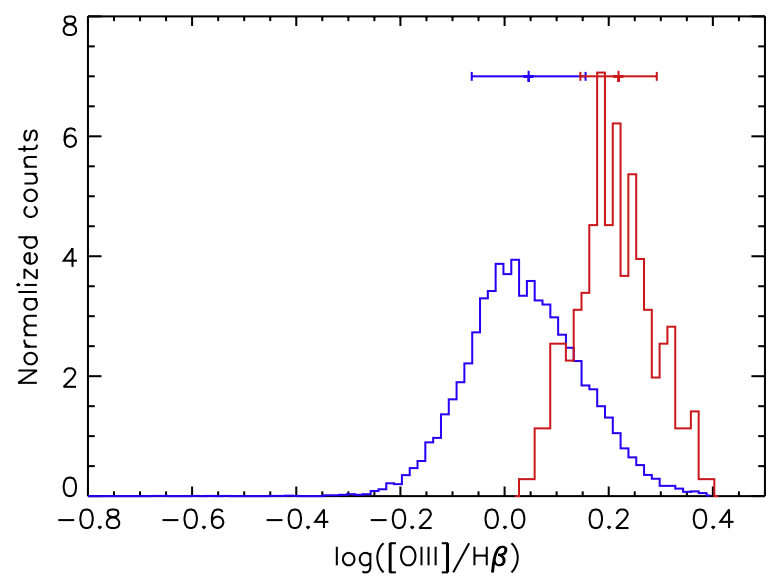

Figure 6. Normalized histograms of $\log \left(\left[\mathrm{O}_{\mathrm{III}}\right] / \mathrm{H} \beta\right.$ values of the map of Arp 220 , so that the area under each histogram is 1 . The histogram in blue shows all $\log ([\mathrm{O} \mathrm{III}] / \mathrm{H} \beta)$ values across the system $(N=18676)$, while the histogram in red shows only the values within the $\mathrm{H} \alpha+\left[\mathrm{N}_{\text {II }}\right]$ bubble feature region $(N=236)$. The mean and standard deviation of each histogram are shown at top.

shown in red. Binned regions are considered part of the ring feature if they fall within $1^{\prime \prime}$ of the center of the ring. The mean $\log ([\mathrm{O} \mathrm{III}] / \mathrm{H} \beta)$ for the entire system is $0.05 \pm 0.11$, or $0.22 \pm$ 0.07 for the bubble region (shown in blue and red, respectively, above the histograms $)$. The $\log \left(\left[\mathrm{O}_{\mathrm{III}}\right] / \mathrm{H} \beta\right)$ values in the ring feature appear to be slightly higher than in the system as a whole, though the difference is not significant at the $1 \sigma$ level.

The value of $\log \left(\left[\mathrm{O}_{\mathrm{III}}\right] / \mathrm{H} \beta\right)$ will be used in conjunction with literature values of $\log \left(\left[\mathrm{N}_{\mathrm{II}}\right] / \mathrm{H} \alpha\right)$ to analyze the gas excitation mechanism in Section 4.2.1.

\subsection{The Bubble in a Multi-wavelength Context}

In this section, we present the first high spatial resolution multi-wavelength maps of Arp 220 combining optical, NIR, and X-ray images. A 3-color image, shown in Figure 7, was made using the optical continuum (HST/WFC3 F621M) image, $\mathrm{H} \alpha+\left[\mathrm{N}_{\mathrm{II}}\right]$ emission image, and the $\mathrm{H}$-band continuum (NICMOS F160W) image. In this image, the blue shows the optical stellar continuum, subject to extinction from dust in the system, especially in the circumnuclear region; the green shows the sum of the $\mathrm{H} \alpha$ and $[\mathrm{N}$ II] emission lines, which can be excited by recent star formation, shocked gas, or AGN excitation; and the red shows a combination of the NIR continuum, which is less affected by dust extinction.

The X-ray structure seen on $\sim 10 \mathrm{kpc}$ scales, which traces the hot gas outflow, appears to be roughly aligned with the P.A. of the bubble feature with respect to the western nucleus (Figure 7). Heckman et al. (1996, Figure 2) reports a P.A. of $315^{\circ}$ for the northwestern plume, while McDowell et al. (2003, Figure 1) report a P.A. of $331^{\circ}$ for the same feature. However, the structure is wide and faint enough that determining an exact P.A. is difficult. In addition, Heckman et al. (1996) report a largescale X-ray structure with a P.A. of $285^{\circ}$, which is inconsistent with the P.A. of the bubble feature. In Figure 7, we show the mid-scale X-ray structure as reported by Heckman et al. (1996). The binned pixel size in the X-ray data $\left(1^{\prime \prime} .5\right)$ is too large to resolve whether the outflow is coming from one of the two nuclei or the $\sim 1 \mathrm{kpc}$ nuclear gas disk. Higher spatial resolution observations reveal a hard X-ray source roughly aligned with the western nucleus (Clements et al. 2002, source X-1).
Therefore, we proceed with the assumption that the X-ray outflows originate in the western nucleus.

The southeastern side of the bubble appears to curve around the western nucleus. This curve is echoed by the NIR emission surrounding the western nucleus, which likely traces the circumnuclear disk (Sakamoto et al. 1999). While little optical emission is seen near the nuclei, due to high dust extinction, there are peaks in the optical emission lines $(\mathrm{H} \alpha+[\mathrm{N} \mathrm{II}]$ and [O III]) roughly coincident with the NIR nuclei. As the images were registered based on the coordinates of SSCs throughout the galaxy, not the nuclei, this alignment is remarkable.

A gap in the ring feature is evident on the side away from the nuclei, which could be due to either high extinction or an actual gap in emission. If the gap is caused by extinction, and the obscuring material contains hot dust, we would expect to see more NIR emission coincident with the gap. The NIR emission does not peak in this area, indicating that this hole in emission is not due to extinction from material containing hot dust. In addition, the mid-scale northwestern X-ray plume is aligned with this gap, suggesting that a stream of hot X-ray emitting gas is clearing a hole in the optical emission line gas, disrupting the process that formed these lines. This hot gas extends for 10-15 kpc on each side of the nucleus (McDowell et al. 2003).

No SSCs appear to be coincident with the bubble feature. Figure 7 shows the locations of the optical and infrared selected SSCs as white crosses (Scoville et al. 1998; Wilson et al. 2006). We discuss this further in Section 4.

\subsection{Kinematics from the NIR}

Engel et al. (2011) studied the NIR kinematics of the circumnuclear region $\left(r \sim 1^{\prime \prime} .5\right)$, centered on the western nucleus, using the $\mathrm{H}_{2}$ 1-0 S(1) line. The velocity structure presented there shows a clear rotational signature, with the rotational axis roughly in the NW/SE direction. The NIR velocity dispersion map is shown in Figure 8, with the optical $\mathrm{H} \alpha+[\mathrm{N}$ II $]$ flux contours overlaid. This map differs slightly from that presented by Engel et al. (2011) in that the instrumental broadening has been corrected for here; thus, the dispersion values are slightly lower than those presented earlier. The velocity dispersion of the $\mathrm{H}_{2}$ is enhanced near the bubble, and reaches a maximum of $250 \mathrm{~km} \mathrm{~s}^{-1}$ at the base of the western arm. The velocity map shows no evidence for a systemic velocity shift at this location, though the rotational axis is not perfectly aligned with the region of enhanced velocity dispersion. This may indicate that the higher velocity dispersion is linked with the bubble feature. If we assume an outflow is launched from a source at the center of the bubble and that the outflow velocity is equal to the peak of the dispersion, then the age of the bubble is $\sim 2.3 \mathrm{Myr}$. If the powering source is in the nucleus, a kinematic age cannot easily be determined. The current kinematic maps do not cover the bubble's full spatial extent. Additional data are necessary to characterize the dynamics of the region.

\subsection{Bubble Energetics}

We estimate the mass and energy in the bubble using $L_{\mathrm{H} \alpha}$, and assuming Case $\mathrm{B}$ recombination and $T=10^{4} \mathrm{~K}$ (Osterbrock 1989). Using the continuum-subtracted $\mathrm{H} \alpha+[\mathrm{N}$ II] image, we find a total integrated flux in the bubble region of $2.6 \times 10^{-14} \mathrm{erg} \mathrm{s}^{-1} \mathrm{~cm}^{-2}$. With the $\mathrm{H} \alpha$ to $\mathrm{H} \alpha+\left[\mathrm{N}_{\mathrm{II}}\right]$ ratio from the bubble region of the Arribas et al. (2001) IFS study 

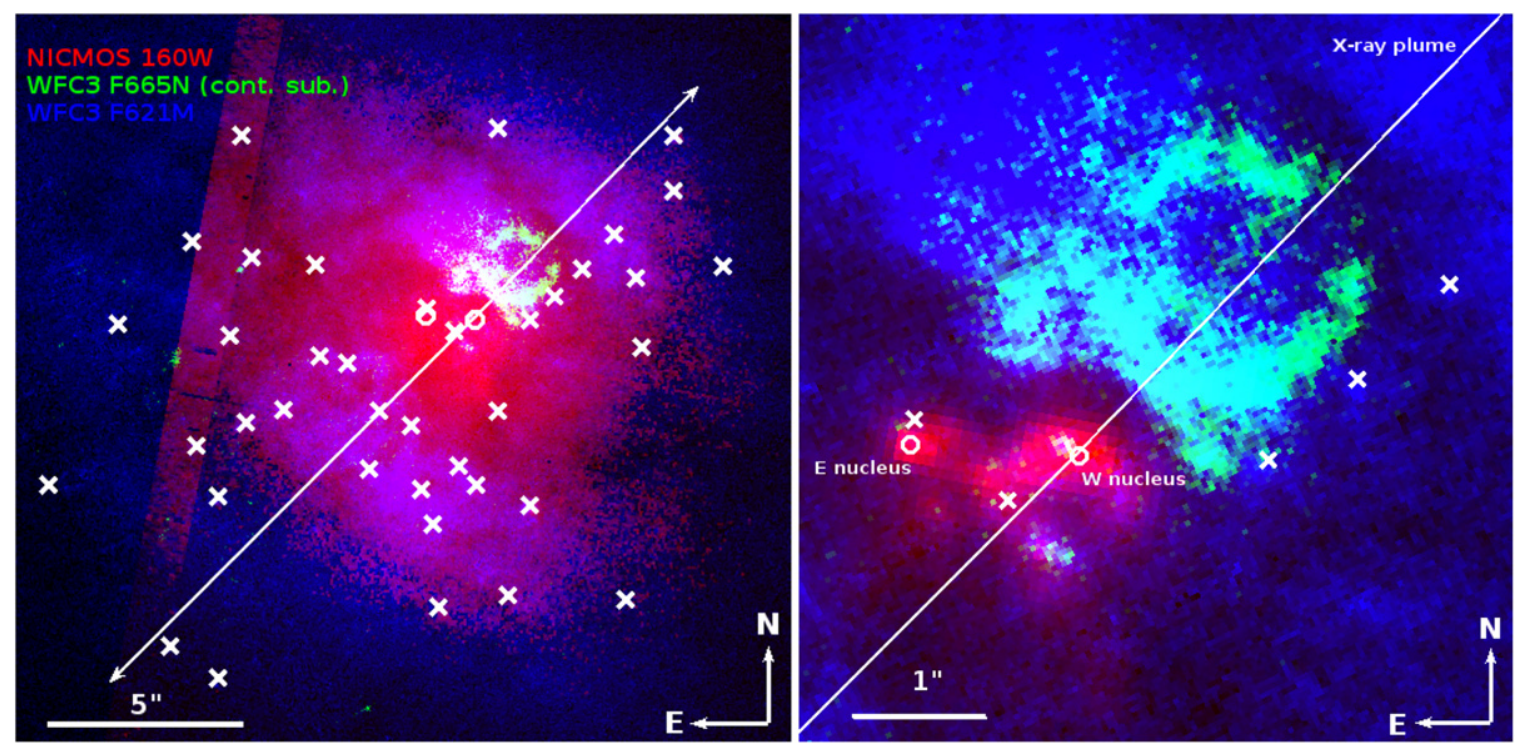

Figure 7. Three color image of Arp 220. The blue image shows the optical continuum (HST/WFC3 F621M), the green shows H $\alpha+[\mathrm{N}$ II] (continuum-subtracted $H S T /$ WFC3 F665N), and the red shows the H-band infrared continuum (HST/NICMOS F160W). The major axis of the soft X-ray structure (white vector) and locations of the super star clusters (SSCs, white crosses) are indicated. The left panel shows the main disk of the system. The scaling of the red image is adjusted to emphasize the faint emission; the chip gap is visible down the left side of the image. The right image shows the nuclear region, including the double nuclei and the H $\alpha+[\mathrm{N}$ II] bubble feature. The peaks in the infrared mark the positions of the two nuclei, and are shown as white circles. The scaling of the red image has been adjusted to show the structure of the strong emission near the nuclei.

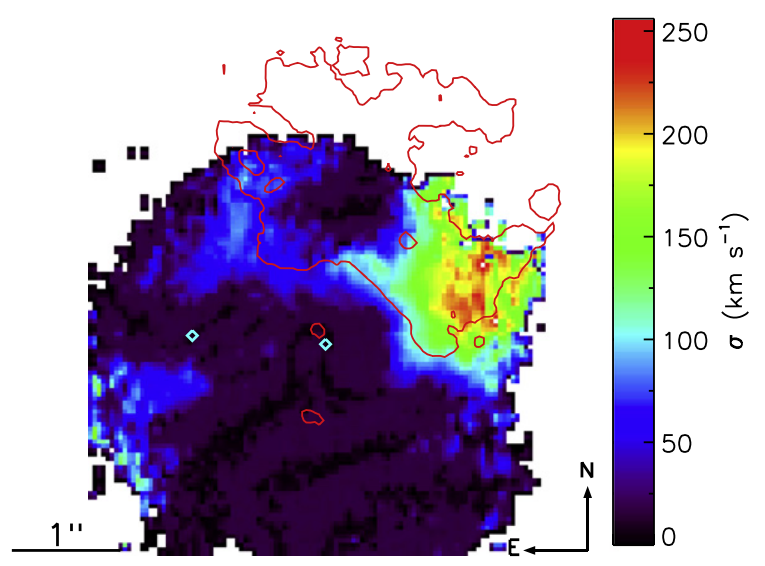

Figure 8. NIR velocity dispersion, calculated from the $\mathrm{H}_{2} 1-0 \mathrm{~S}(1)$ line and corrected for instrumental broadening. Contours show the $7 \sigma \mathrm{H} \alpha+[\mathrm{N}$ II] flux, smoothed for display purposes. The nuclei are marked as cyan diamonds. North is up and east is left.

$\left(\frac{\mathrm{H} \alpha}{\mathrm{H} \alpha+[\mathrm{N} \mathrm{II}]}=0.37\right)$, we calculate an ionized gas mass of $2.5 \times 10^{7} M_{\odot} n_{e}^{-1}$.

The $\mathrm{H} \alpha$ flux used here has not yet been corrected for extinction. We can derive the extinction in the bubble region via the Balmer decrement, by taking the ratio of the $\mathrm{H} \alpha$ to $\mathrm{H} \beta$ maps and comparing this value to 2.86 , the theoretical ratio under Case B recombination. This calculation is very sensitive to the continuum subtraction for $\mathrm{H} \beta$ (see Section 2.1.1), but we can derive a range of acceptable extinction values based on the error in the continuum subtraction scaling factor. The mean continuum scaling factor for $\mathrm{H} \beta, 0.42$, gives $E(B-V)=0.11$, though a range in $E(B-V)$ of $0.00-0.48$ is also consistent. Given the mean extinction, we derive a dereddened ionized gas mass of $M_{\text {ion }}=3.2 \times 10^{7} M_{\odot} n_{e}^{-1}$, with a range of $(2.5-7.8) \times$ $10^{7} M_{\odot} n_{e}^{-1}$. We adopt the mass derived from the mean value of the $\mathrm{H} \beta$ continuum scaling factor in the following discussion.

The $\left[\mathrm{S}_{\mathrm{II}}\right]$ doublet ratio $6317 \AA / 6331 \AA$ can be used to constrain the electron density of the ionized gas. The MPAJHU SDSS DR7 data product catalog 9 gives a ratio of 1.46, which places $n_{e}$ in the lower density limit (Osterbrock 1989), or less than $\sim 10^{2} \mathrm{~cm}^{-3}$. Previous work on the ionized bubble in NGC 3079 (Veilleux et al. 1994; Cecil et al. 2001) constrained $n_{e}$ in that system to $5-100 \mathrm{~cm}^{-3}$. Rupke \& Veilleux (2013b) adopt $n_{e}=10 \mathrm{~cm}^{-3}$ for their analysis of the ionized winds in a sample of ULIRGs, consistent with the NGC 3079 limits. We adopt $n_{e}=10 \mathrm{~cm}^{-3}$ here to facilitate comparison with the literature. Assuming the upper limit of $n_{e}=10 \mathrm{~cm}^{-3}$ gives $M_{\text {ion }}=3.2 \times 10^{6} M_{\odot}$. Rupke \& Veilleux (2013b, Table 6) find ionized gas masses in their sample of ULIRG winds in the range $10^{6.93-8.07} M_{\odot}$, so the Arp 220 bubble mass is similar to these other ULIRG outflows.

We estimate the kinetic energy in an expanding shell by assuming that the observed velocity dispersion in $\mathrm{H}_{2}$ represents the shell velocity, or $v_{\text {shell }}=250 \mathrm{~km} \mathrm{~s}^{-1}$. We find a kinetic energy of $2.0 \times 10^{54} \mathrm{erg}$. Given a dynamical age of $2.3 \mathrm{Myr}$ (Section 3.4) and assuming constant energy injection, we calculate an energy injection rate from the ionized gas mass of $2.8 \times 10^{40} \mathrm{erg} \mathrm{s}^{-1}$.

We are only observing the ionized gas and the neutral gas may be a substantial fraction of the total gas mass in the bubble feature. To include a correction for the neutral hydrogen, we used the measurements of Mundell et al. (2001), who measured hydrogen absorption against the continuum in $1.4 \mathrm{GHz}$ observations. The structure they identify as spur $\mathrm{T}$ is coincident with the bubble feature, though they are unable to measure significant $\mathrm{H}$ absorption in this region. They place an upper limit of $N_{\mathrm{H}}<1.3 \times 10^{20} T_{s}(\mathrm{~K}) \mathrm{cm}^{-2}$. With their assumption that $T_{s}=100 \mathrm{~K}$, that gives an upper limit of

9 http://www.mpa-garching.mpg.de/SDSS/DR7/ 
$N_{\mathrm{H}}<1.3 \times 10^{22} \mathrm{~cm}^{-2}$, and an upper limit on the total neutral hydrogen mass in the bubble region of $1.2 \times 10^{8} M_{\odot}$. Using the same velocity assumptions as for the ionized gas, the upper limit of kinetic energy in the neutral gas is $3.8 \times 10^{55} \mathrm{erg}$, and the upper limit on the energy injection rate is $5.3 \times 10^{41} \mathrm{erg} \mathrm{s}^{-1}$.

The total neutral+ionized mass in the bubble feature is thus $M<1.2 \times 10^{8} M_{\odot}$, the total kinetic energy is $\mathrm{KE}<4.0 \times$ $10^{55} \mathrm{erg}$, and the energy injection rate is $d E / d t \sim 5.6 \times$ $10^{41} \mathrm{erg} \mathrm{s}^{-1}$. We compare these rates to possible sources in the following section.

\section{ORIGIN OF THE BUBBLE}

Given the bubble's position and morphology, there are two possible points of origin. First, it could be the product of a jet or outflow originating in one of the two nuclei or the molecular disk immediately surrounding them. Alternatively, the bubble could originate from a source at its center. Either a massive star cluster or a microquasar (i.e., an ultraluminous X-ray source, ULX) could drive a traditional expanding superbubble. Given that the nuclei are deeply embedded in dense gas and are more distant, the energies necessary to drive an outflow from the nuclear disk versus from the bubble's center are very different. We lay out the possibilities in Sections 4.1 and 4.2, and discuss and compare them further in Section 4.3.

\subsection{Nuclear Origins}

The first possibility is that the bubble is being driven from a nucleus or the circumnuclear region, either from an $\operatorname{AGN}(\mathrm{s})$ or from a strong nuclear starburst.

\subsubsection{Jet or Outflow from an AGN}

The observed morphology of the bubble feature matches that produced in some AGN jet simulations. Sutherland \& Bicknell (2007) modeled the interaction of a light supersonic jet with the inhomogeneous and turbulently supported ISM in an earlytype galaxy. Their simulated ISM followed a lognormal singlepoint density distribution and a power-law density structure, with an average jet/ISM number density ratio of $\sim 10^{-2}$. The jet had a kinetic luminosity of $\sim 10^{43} \mathrm{erg} \mathrm{s}^{-1}$, and radiative cooling was implemented via the MAPPINGS photoionization and shock code (Sutherland \& Dopita 1993).

The evolution of the interaction goes through several stages, including the development of a shocked bubble as the jet interacts with the ISM followed by a breakthrough of the jet through the outer boundary of the bubble. The simulation midplane density slices post-breakout (their Figure 2, and reproduced here, in Figure 9) resemble the Arp 220 bubble. However, because this is a generic jet/ISM simulation, this is an idealized comparison; in particular, the ISM of Arp 220 is likely to be denser than that assumed here. Nonetheless, the morphological similarities suggest the possible presence of an AGN and its associated outflow.

The proximity of the nuclei to each other $(360 \mathrm{pc})$ suggests that they should be subject to strong gravitational interactions, but clear observational evidence of an AGN has been sparse. Previous X-ray studies (Rieke 1988; Dermer et al. 1997; Iwasawa 1999; Iwasawa et al. 2001; Clements et al. 2002) ruled out a bright hard X-ray source in the nucleus, which indicates that any AGN must be highly obscured, with a lower limit on the nuclear column density of $10^{25} \mathrm{~cm}^{-2}$. However, evidence for at least one highly obscured AGN in Arp 220 has

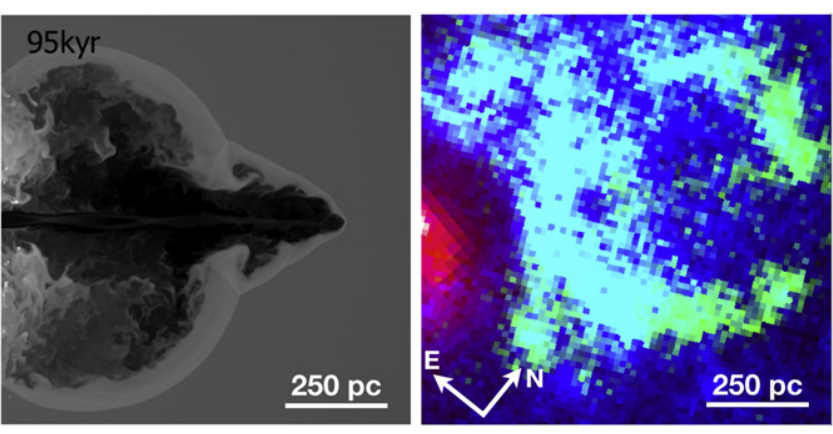

Figure 9. Left: last panel of Figure 2 from Sutherland \& Bicknell (2007), reproduced here with permission. These simulations model the interaction of a supersonic jet with an inhomogenous ISM. The figure shows the $95 \mathrm{kyr}$ timestep of the mid-plane density slices of the simulation, with darker colors representing less dense material. The panel is $1 \mathrm{kpc}$ on a side. Right: three-color image of the bubble feature, as in the right panel of Figure 7, rotated and zoomed for comparison with the simulation. The western nucleus is centered on the left side of the panel and the opening in the bubble is centered on the right side of the panel. This panel is also $1 \mathrm{kpc}$ on a side.

been mounting in recent years. A maser (Aalto et al. 2009) and a rotating massive molecular disk (Downes \& Eckart 2007) have been found, and Contini (2013) find evidence of an AGN in their modeling of the nuclear optical and infrared spectra. Paggi et al. (2013) find strong Fe-K $\alpha$ line emission in the vicinity of the two nuclei, concentrated most strongly on the western nucleus. From their broadband X-ray data, they estimate lower limits on the AGN bolometric luminosities of $5.2 \times 10^{43} \mathrm{erg} \mathrm{s}^{-1}$ (western nucleus) and $2 \times 10^{42} \mathrm{erg} \mathrm{s}^{-1}$ (eastern nucleus). New ALMA observations (Wilson et al. 2014) show a luminosity surface density in excess of $10^{14} L_{\odot} \mathrm{kpc}^{-2}$ at the western nucleus, sufficiently bright to require the presence of a hot starburst, a Compton thick AGN, or both.

As shown in Section 3.5, the kinetic energy input needed to form the bubble is $d E / d t=5.6 \times 10^{41} \mathrm{erg} \mathrm{s}^{-1}$ (upper limit, ionized+neutral medium). Taking this as a ratio with respect to the bolometric luminosity of the suspected AGN in the western nucleus gives an energy deposition rate of $d E / d t L_{\mathrm{AGN}}=1.1 \%$, which is an order of magnitude higher than other observed (Rupke \& Veilleux 2013b) or modeled AGN energy feedback rates (Hopkins \& Elvis 2010). However, these models are dependent on the fraction of gas in the hot ISM and are uncertain enough to encompass our observed value.

The evidence for an obscured AGN driving the bubble is favorable but not absolute. The morphological similarities to models and the energetics lend support to the theory that this bubble is formed by the interaction of a jet or outflow from an AGN with the galaxy's ISM. This is further supported by the large-scale X-ray structure of the galaxy, which is remarkably well aligned with not just the bubble feature, but also the gap in the side of the bubble away from the nuclei (Figure 7). However, the western nucleus (the most likely AGN host) has a gas accretion disk that is misaligned with the supposed direction of the jet and bubble. This is not definitive evidence against an AGN outflow, because the observed gas disk has a scale of $100 \mathrm{pc}$ and any inner accretion disk may have a different orientation. Additionally, the outflow velocities in this system are on the low end for typical AGNs (Rupke et al. 2005a; Tremonti et al. 2007), but may be consistent with velocities from low-luminosity AGNs (Christopoulou et al. 1997; Kewley et al. 2006b; Hicks et al. 2013). We view 
an AGN jet or outflow as a plausible hypothesis for this bubble's origin.

\subsubsection{Collimated Outflow from a Nuclear Starburst}

The prevailing explanation for the large-scale superwind structure as seen in X-rays is a massive starburst concentrated near the nuclei (Heckman et al. 1996). Smith et al. (1998) used VLBI continuum imaging to resolve a strong, compact $\mathrm{OH}$ maser near the western nucleus into a number of radio supernovae within a $75 \times 150 \mathrm{pc}$ region. Using the lifetime of these objects, they estimate a nuclear star formation rate of $50-800 M_{\odot} \mathrm{yr}^{-1}$. Such high levels of star formation in the confines of the nuclear gas disk could potentially drive a starburst wind. If the starburst wind is able to break through the gas disk, the dense gas in the disk will further direct the outflow along the maximum pressure gradient.

In Section 3.5, we showed that the kinetic energy input needed to form the bubble is $d E / d t=5.6 \times 10^{41} \mathrm{erg} \mathrm{s}^{-1}$ (upper limit, ionized+neutral medium). If we assume a SFR of $240 M_{\odot} \mathrm{yr}^{-1}$ (equal to the global SFR and well within the nuclear SFR range), we can compare the energy in the wind to the mechanical luminosity in the nuclear starburst. Following Rupke \& Veilleux (2013b), we calculate the mechanical luminosity in a continuous starburst using Starburst99 models from Leitherer et al. (1999). We apply a correction for a Salpeter IMF, with a lower mass cut at $0.1 M_{\odot}$. Thus, the energy deposition rate is $d E / d t / d E / d t_{\mathrm{SB} 99}=0.8 \%$ (upper limit). This is in line with that observed in other starburstdominated ULIRGs (3\%-13\%, Rupke \& Veilleux 2013b). It is thus also possible that the bubble feature is driven by the strong nuclear starburst.

\subsection{Off-nuclear Origins}

The bubble may originate from a source at its center rather than from the nucleus. It could be the product of a massive star cluster, or of a microquasar or ULX object.

\subsubsection{Massive Star Cluster}

Structures morphologically similar to the Arp 220 bubble feature, known as superbubbles, are believed to be formed by supernovae winds from a massive star cluster pushing out into the surrounding ISM (Cash et al. 1980). The Arp 220 bubble feature demonstrates some evidence consistent with a classical superbubble, but lacks evidence of a sufficiently massive star cluster driving the outflow.

The Arp 220 bubble shows evidence of shock excitation, which is consistent with a supernovae-driven outflow from a massive young cluster. Such outflows shock as they encounter the surrounding ISM and produce an increasing ratio of $[\mathrm{N}$ II] to $\mathrm{H} \alpha$ emission at a fixed value of $\log \left(\left[\mathrm{O}_{\mathrm{III}}\right] / \mathrm{H} \beta\right.$ ) (Baldwin et al. 1981; Kewley et al. 2001, 2006b). The value of $\log$ $([\mathrm{O}$ III $] / \mathrm{H} \beta)$ is fairly constant across the center of the galaxy at $0.22 \pm 0.07$ (see Section 3.2, $0.05 \pm 0.11$ for the galaxy as a whole $)$. The value of $\log ([\mathrm{N} \mathrm{II}] / \mathrm{H} \alpha)$ at the maximum starburst line, or the value at which shock heating is thought to begin to dominate (Kewley et al. 2001), is $-0.16 \pm 0.04$ for this value of $\log \left(\left[\mathrm{O}_{\mathrm{III}}\right] / \mathrm{H} \beta\right)$ (though there are metallicity effects; see, e.g., Dopita et al. 2000; Denicoló et al. 2002; Kewley \& Dopita 2002; Kewley et al. 2006b). Though we cannot separate the $[\mathrm{N}$ II $]$ and $\mathrm{H} \alpha$ emission to calculate a ratio with our data, Arribas et al. (2001) show optical IFU spectroscopy data taken with the INTEGRAL instrument on the William Herschel telescope. Their data are lower spatial resolution (fiber diameter $\sim 0$ ". 9, $R \sim 2000$ ) and they identify three gas components via spectral line profile decomposition. Spatially, our bubble feature is associated with their component B. They find an average value of $\log \left(\left[\mathrm{N}_{\text {II }}\right] / \mathrm{H} \alpha\right)$ of $0.48 \pm 0.17$ for this component. This is consistent with shocked gas in a shell surrounding a massive star cluster that is driving the outflow.

Although our feature resembles typical massive star cluster superbubbles, no massive star clusters are visible in the center of the bubble (Figure 7). Examination of both the optical continuum image (WFC3-UVIS F621M) and the H-band image (NICMOS F160W) reveals that the flux interior to the ring feature is high enough that a $10^{6} M_{\odot}$ cluster would be hidden by the background. To determine this limit, we measured the flux of other $10^{6} M_{\odot}$ clusters outside the bubble as identified by Wilson et al. (2006, clusters 2, 3, 4, 5, 11, and 12). The mean flux for a $10^{6} M_{\odot}$ cluster is $3.52 \pm$ $1.50 \times 10^{-18} \mathrm{erg} \mathrm{cm}^{-2} \mathrm{~s}^{-1} \AA^{-1}$ for WFC3 F621M and $1.77 \pm$ $0.86 \times 10^{-18} \mathrm{erg} \mathrm{cm}^{-2} \mathrm{~s}^{-1} \AA^{-1}$ for NICMOS F160W, using an aperture of radius $0^{\prime \prime} .17$.

Analysis of the energetics provides additional evidence against an outflow driven by a massive young cluster at the bubble center. The mechanical luminosity produced by a $10^{6} M_{\odot}$ cluster $\left(\sim 10^{40} \mathrm{erg} \mathrm{s}^{-1}\right.$ Leitherer et al. 1999) is a factor of $\sim 100$ below the energy injection rate $\left(\sim 10^{42} \mathrm{erg} \mathrm{s}^{-1}\right)$ we derived for the bubble. Thus, while we cannot rule out the existence of an undiscovered cluster smaller than $10^{6} M_{\odot}$, it is unlikely that it is the origin of the bubble feature.

\subsubsection{ULX Bubble}

ULX are compact X-ray sources whose luminosities exceed the Eddington luminosity of an isotropically emitting stellarmass object. By definition, they are located away from the nucleus of their host galaxy. The central excitation source is still unknown, though their observed variability has been used to hypothesize these as accreting stellar- or intermediate-mass black holes (Colbert \& Mushotzky 1999; Kubota et al. 2001). They can exhibit either soft or hard X-ray spectra, and an individual ULX can transition between the two spectral modes (Kubota et al. 2001), presumably due to variability in accretion onto the central source. Many of these objects in the local universe (within $10 \mathrm{Mpc}$ ) are associated with large ionized nebulae up to several hundred pc in diameter (Pakull \& Mirioni 2002; Cseh et al. 2012; Dopita et al. 2012). These nebulae often show bright $\mathrm{H} \alpha$ bubbles, with the X-ray source either within the bubble or on one edge. Optically, they are generally associated with strong narrow-line He II $\lambda 4686 \AA$ emission. This high-ionization line is not readily created by normal O-stars, though broad He II lines are observed in WolfRayet stars.

The bubble feature in Arp 220 resembles these ULX bubbles. Its physical extent, $600 \mathrm{pc}$ in diameter, is roughly the same size as the known ULX bubbles. It is also coincident with a bright $\left(L_{0.3-10.0 \mathrm{keV}} \sim 10^{39} \mathrm{erg} \mathrm{s}^{-1}\right)$ soft X-ray peak observed by Chandra (Clements et al. 2002, their X-3; Figure 10). However, while the X-ray sources associated with the local ULX bubbles are generally compact, Arp 220-X-3 appears to be extended, or embedded in the same extended emission that surrounds the nuclei. Clements et al. (2002) report an extent of $2^{\prime \prime}$, shown as the yellow circle in Figure 10. No data exist on possible variability of this source, or of 


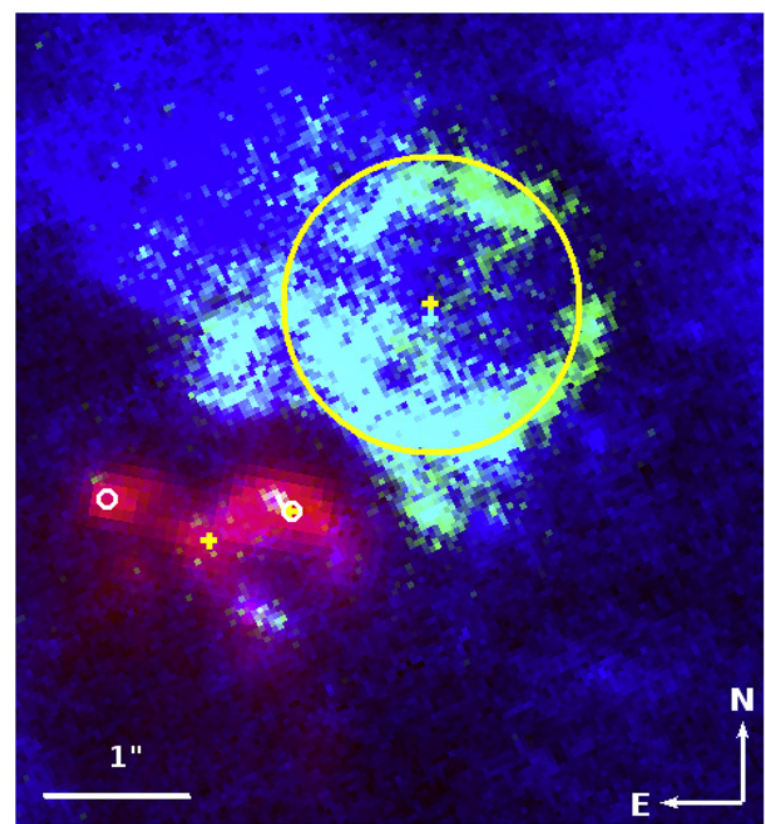

Figure 10. Three-color image of the central bubble. Background is the same as Figure 7, right panel. Chandra-identified X-ray sources, from Clements et al. (2002), are marked as yellow crosses, and the two NIR nuclei (Scoville et al. 1998, see Section 2.3) are marked as white circles. From east to west, the three sources shown are their X-4, X-1 (nuclear), and X-3 (X-2 is out of the field of view). The $\mathrm{X}$-ray source coordinates have been shifted so as to align their $\mathrm{X}-1$ nuclear source with the western NIR nucleus; thus the western nucleus marker is obscured. X-1 and X-4 are both unresolved hard X-ray peaks, while X-3 is the peak of extended soft X-ray emission (extent of $2^{\prime \prime}$, shown as the yellow circle).

narrow-line He II emission. Finally, given the alignment of the bubble feature with the large-scale X-ray outflow, it is unlikely that the bubble feature has an origin unrelated to this structure. The large-scale outflow is bipolar and thus likely nuclear in origin. Further study is needed to explore whether this X-ray source is indeed a ULX with an affiliated nebula, or whether the X-ray emission is from hot gas filling the bubble created via one of the mechanisms described above.

\subsection{Bubble Origins, Summarized}

We have explored four possibilities for the origin of the bubble: an outflow from an obscured AGN, an outflow from high levels of star formation within the nuclear gas disk, a traditional superbubble surrounding an undiscovered star cluster at the bubble's center, or a ULX bubble.

Given the alignment of the nuclei, bubble feature, and largescale (biconal) outflow structure, as well as energetics arguments, it is less likely that the feature is caused by an off-nuclear source unrelated to the outflow. While a chance alignment of a ULX superbubble is possible, and warrants further study, it is more likely that an outflow or jet from the nuclear region is driving both the bubble and the outflow.

Out of the two nuclear origin scenarios, we cannot distinguish between an AGN jet or outflow or a starburst outflow. The low luminosity of the suspected AGN in the western nucleus $\left(\sim 10^{43} \mathrm{erg} \mathrm{s}^{-1}\right)$ and the high levels of star formation within the inner nuclear gas disks around both nuclei would each provide a similar rate of energy injection, either of which would be sufficient to drive the bubble feature. It is also possible that both mechanisms are contributing to the outflow driving the bubble.

\section{CONCLUSIONS}

We have resolved the near-nuclear $\mathrm{H} \alpha+\left[\mathrm{N}_{\text {II }}\right]$ emission peak into a bubble feature using HST/WFC3 narrowband photometry. Our new multi-wavelength imaging maps connect this feature to both the nuclear molecular gas disk and large-scale outflows. NIR IFS data show an increase in velocity dispersion at the base of the bubble, while the optical IFS data show that the emission in this region is dominated by shock excitation. This strongly indicates that the bubble is formed by an interaction of a jet or outflow, originating in or near the nucleus, with the surrounding ISM. This outflow shocks as it propagates into the surrounding galaxy and is connected spatially with the large-scale outflow that extends for multiple kpc from the galaxy. We discuss four possibilities of the origin of the bubble and find it most plausible that the bubble originates within the inner $\sim 100 \mathrm{pc}$ around the nuclei. Either an obscured AGN or the observed high level of nuclear star formation could power the observed feature. Further work is needed to differentiate between these two possibilities.

Some of the data presented in this paper were obtained from the Mikulski Archive for Space Telescopes (MAST). STScI is operated by the Association of Universities for Research in Astronomy, Inc., under NASA contract NAS5-26555. Support for MAST for non-HST data is provided by the NASA Office of Space Science via grant NNX13AC07G and by other grants and contracts. K.E.L. would also like to acknowledge the SWOOP writing retreat and its participants for useful feedback.

\section{APPENDIX}

Narrowband imaging of $\left[\mathrm{S}_{\mathrm{II}}\right] \lambda \lambda 6717,6731 \AA$, in conjunction with similar imaging of $\mathrm{H} \alpha$, has been used previously on dwarf galaxies (Calzetti et al. 2004) to separate photoionized from non-photoionized gas, and hence to evaluate photoionized gas oxygen abundances and non-photoionized gas distributions. One of the primary motivations for obtaining the Arp 220 HST dataset presented in this paper was to apply this same technique on a late-stage merger. Unfortunately, the $\left[\mathrm{S}_{\mathrm{II}}\right]$ emission line flux could not be recovered from the F680N filter, due to both complicating factors specific to the combination of the redshift of Arp 220 and this filter, and to the shallowness of the image. In this appendix, we describe the reduction process for this filter and suggest ways to improve the process.

The F680N filter, which contains the $[\mathrm{S} \mathrm{II}]$ lines, is also contaminated by some of the $\mathrm{H} \alpha+[\mathrm{N}$ II $]$ line complex, which falls on the blue cutoff of the F680N bandpass (Figure 2). Because the bandpass cutoff falls in the middle of the intruding line complex, we cannot simply subtract the flux-calibrated $\mathrm{H} \alpha$ $+\left[\mathrm{N}_{\mathrm{II}}\right]$ image from the $[\mathrm{S}$ II $]$ image. Instead, we must estimate the percentage of the contaminating lines that fall in the bandpass. We convolved the SDSS spectrum with the theoretical throughputs of the F665N and the F680N filters obtained from the synphot PyRAF ${ }^{10}$ package to calculate the contribution of the flux of these lines. Roughly half the flux in the $\mathrm{H} \alpha+[\mathrm{N}$ II $]$ line complex in the SDSS spectrum falls in the

\footnotetext{
${ }^{10}$ STSDAS and PyRAF are products of the Space Telescope Science Institute, which is operated by AURA for NASA.
} 
F680N bandpass, though the exact contribution depends on the width and velocity of the lines, metallicity, and gas excitation mechanism. This will likely vary across the face of the galaxy, and introduces additional uncertainties.

Changes in the line of sight velocity may shift the $\mathrm{H} \alpha+[\mathrm{N}$ II] lines in or out of the F680N bandpass, altering the scaling factor used to remove the flux contributed by these lines. This error was estimated by red and blue shifting the SDSS spectrum by $500 \mathrm{~km} \mathrm{~s}^{-1}$ along the line of sight. At the velocity extremes, the flux contribution from the $\mathrm{H} \alpha+[\mathrm{N}$ II] lines complex ranges from $35 \%$ to $80 \%$ of the flux in these lines. The additional error in flux is included in the $1 \sigma$ errors on the [S II] image in Table 1.

However, subtracting $50 \%$ of the $\mathrm{H} \alpha+\left[\mathrm{N}_{\text {II }}\right]$ flux from the continuum-subtracted $\left[\mathrm{S}_{\mathrm{II}}\right]$ image produced oversubtracted images with negative flux values near the center of the galaxy, clear artifacts of the $\mathrm{H} \alpha+\left[\mathrm{N}_{\mathrm{II}}\right]$ subtraction. This is likely due to high extinction in the central regions. We explored several avenues for improving continuum subtraction in this filter, all of which were ultimately unsuccessful at producing robust detections of $[\mathrm{S}$ II] . This is likely due to the intrinsic faintness of the $[\mathrm{S}$ II $]$ lines, which are only $10 \%$ of the flux in $\mathrm{H} \alpha+[\mathrm{N} \mathrm{II}]$ on average, and the short exposure time in the F680N filter.

To make use of the $\left[\mathrm{S}_{\mathrm{II}}\right]$ data, we would require a longer exposure time in the F680N filter to allow us to probe the fainter average $\left[\mathrm{S}_{\mathrm{II}}\right]$ emission. Alternatively, separate high spatial resolution map of both $[\mathrm{N}$ II] and $\mathrm{H} \alpha$ flux and velocity structure would allow us to more robustly correct the $[\mathrm{S} \mathrm{II}]$ image for the $[\mathrm{N} \mathrm{II}]$ contamination, and hence recover more $\left[\mathrm{S} \mathrm{II}^{\mathrm{II}}\right]$ flux. Without either of these, the current $\left[\mathrm{S}_{\mathrm{II}}\right]$ data are inadequate for our purposes. Future projects that attempt this type of analysis should be especially careful to obtain deep images of the fainter emission lines.

\section{REFERENCES}

Aalto, S., Wilner, D., Spaans, M., et al. 2009, A\&A, 493, 481 Arp, H. 1966, ApJS, 14, 1

Arribas, S., Colina, L., \& Clements, D. 2001, ApJ, 560, 160

Avila, R. J., Hack, W. J., \& STScI AstroDrizzle Team 2012, BAAS, 220, $\# 135.13$

Baan, W. A., \& Haschick, A. D. 1995, ApJ, 454, 745

Baldwin, J. A., Phillips, M. M., \& Terlevich, R. 1981, PASP, 93, 5

Barnes, J. E., \& Hernquist, L. 1996, ApJ, 471, 115

Calzetti, D., Harris, J., Gallagher, J. S., III, et al. 2004, AJ, 127, 1405

Cash, W., Charles, P., Bowyer, S., et al. 1980, ApJL, 238, L71

Cecil, G., Bland-Hawthorn, J., Veilleux, S., \& Filippenko, A. V. 2001, ApJ, 555,338

Christopoulou, P. E., Holloway, A. J., Steffen, W., et al. 1997, MNRAS, 284,385

Clements, D. L., McDowell, J. C., Shaked, S., et al. 2002, ApJ, 581, 974

Colbert, E. J. M., \& Mushotzky, R. F. 1999, ApJ, 519, 89

Colina, L., Arribas, S., \& Clements, D. 2004, ApJ, 602, 181

Contini, M. 2013, MNRAS, 429, 242

Cresci, G., Mannucci, F., Della Valle, M., \& Maiolino, R. 2007, A\&A, 462, 927

Cseh, D., Corbel, S., Kaaret, P., et al. 2012, ApJ, 749, 17

de Vaucouleurs, G., de Vaucouleurs, A., Corwin, H. G., Jr., et al. 1991, Third Reference Catalogue of Bright Galaxies (Berlin: Springer)

Denicoló, G., Terlevich, R., \& Terlevich, E. 2002, MNRAS, 330, 69

Dermer, C. D., Bland-Hawthorn, J., Chiang, J., \& McNaron-Brown, K. 1997, ApJL, 484, L121

Di Matteo, T., Springel, V., \& Hernquist, L. 2005, Natur, 433, 604

Dopita, M. A., Kewley, L. J., Heisler, C. A., \& Sutherland, R. S. 2000, ApJ, 542,224

Dopita, M. A., Payne, J. L., Filipović, M. D., \& Pannuti, T. G. 2012, MNRAS, 427, 956

Downes, D., \& Eckart, A. 2007, A\&A, 468, L57

Downes, D., \& Solomon, P. M. 1998, ApJ, 507, 615
Eisenhauer, F., Abuter, R., Bickert, K., et al. 2003, Proc. SPIE, 4841, 1548

Emonts, B. H. C., Piqueras-López, J., Colina, L., et al. 2014, A\&A, 572, A40 Engel, H., Davies, R. I., Genzel, R., et al. 2011, ApJ, 729, 58

Farrah, D., Afonso, J., Efstathiou, A., et al. 2003, MNRAS, 343, 585

Forbes, D. A., Polehampton, E., Stevens, I. R., Brodie, J. P., \& Ward, M. J. 2000, MNRAS, 312, 689

Heckman, T. M. 2003, RMxAA, 17, 47

Heckman, T. M., Armus, L., \& Miley, G. K. 1987, AJ, 93, 276

Heckman, T. M., Armus, L., \& Miley, G. K. 1990, ApJS, 74, 833

Heckman, T. M., Dahlem, M., Eales, S. A., Fabbiano, G., \& Weaver, K. 1996, ApJ, 457, 616

Heckman, T. M., Lehnert, M. D., Strickland, D. K., \& Armus, L. 2000, ApJS, 129,493

Hibbard, J. E., Vacca, W. D., \& Yun, M. S. 2000, AJ, 119, 1130

Hicks, E. K. S., Davies, R. I., Maciejewski, W., et al. 2013, ApJ, 768, 107

Hong, S., Calzetti, D., \& Dickinson, M. 2014, PASP, 126, 79

Hopkins, P. F., \& Elvis, M. 2010, MNRAS, 401, 7

Hopkins, P. F., \& Hernquist, L. 2006, ApJS, 166, 1

Hopkins, P. F., Hernquist, L., Cox, T. J., et al. 2005, ApJ, 630, 705

Iwasawa, K. 1999, MNRAS, 302, 96

Iwasawa, K., Matt, G., Guainazzi, M., \& Fabian, A. C. 2001, MNRAS, 326, 894

Joseph, R. D., \& Wright, G. S. 1985, MNRAS, 214, 87

Kauffmann, G., Heckman, T. M., Tremonti, C., et al. 2003, MNRAS, 346, 1055

Kenney, J. D. P., \& Yale, E. E. 2002, ApJ, 567, 865

Kewley, L. J., \& Dopita, M. A. 2002, ApJS, 142, 35

Kewley, L. J., Dopita, M. A., Sutherland, R. S., Heisler, C. A., \& Trevena, J. 2001, ApJ, 556, 121

Kewley, L. J., Geller, M. J., \& Barton, E. J. 2006a, AJ, 131, 2004

Kewley, L. J., Groves, B., Kauffmann, G., \& Heckman, T. 2006b, MNRAS, 372, 961

Kubota, A., Mizuno, T., Makishima, K., et al. 2001, ApJL, 547, L119

Leitherer, C., Schaerer, D., Goldader, J. D., et al. 1999, ApJS, 123, 3

Martin, C. L. 2005, ApJ, 621, 227

Martin, C. L. 2006, ApJ, 647, 222

McDowell, J. C., Clements, D. L., Lamb, S. A., et al. 2003, ApJ, 591, 154

Mihos, J. C., \& Hernquist, L. 1996, ApJ, 464, 641

Mundell, C. G., Ferruit, P., \& Pedlar, A. 2001, ApJ, 560, 168

Oey, M. S., Parker, J. S., Mikles, V. J., \& Zhang, X. 2003, AJ, 126, 2317

Osterbrock, D. E. 1989, Astrophysics of Gaseous Nebulae and Active Galactic Nuclei (Mill Valley, CA: University Science Books)

Paggi, A., Fabbiano, G., Risaliti, G., Wang, J., \& Elvis, M. 2013, arXiv: 1303.2630

Pakull, M. W., \& Mirioni, L. 2002, arXiv:astro-ph/0202488

Rich, J. A., Dopita, M. A., Kewley, L. J., \& Rupke, D. S. N. 2010, ApJ, 721,505

Rieke, G. H. 1988, ApJL, 331, L5

Rupke, D. S., Veilleux, S., \& Sanders, D. B. 2002, ApJ, 570, 588

Rupke, D. S., Veilleux, S., \& Sanders, D. B. 2005a, ApJ, 632, 751

Rupke, D. S., Veilleux, S., \& Sanders, D. B. 2005b, ApJS, 160, 87

Rupke, D. S., Veilleux, S., \& Sanders, D. B. 2005c, ApJS, 160, 115

Rupke, D. S. N., \& Veilleux, S. 2011, ApJL, 729, L27

Rupke, D. S. N., \& Veilleux, S. 2013a, ApJL, 775, L15

Rupke, D. S. N., \& Veilleux, S. 2013b, ApJ, 768, 75

Sakamoto, K., Aalto, S., Combes, F., Evans, A., \& Peck, A. 2014, ApJ, 797, 90

Sakamoto, K., Scoville, N. Z., Yun, M. S., et al. 1999, ApJ, 514, 68

Sanders, D. B., Soifer, B. T., Elias, J. H., et al. 1988, ApJ, 325, 74

Scoville, N., Sheth, K., Walter, F., et al. 2015, ApJ, 800, 70

Scoville, N. Z., Evans, A. S., Dinshaw, N., et al. 1998, ApJL, 492, L107

Scoville, N. Z., Yun, M. S., \& Bryant, P. M. 1997, ApJ, 484, 702

Smith, H. E., Lonsdale, C. J., Lonsdale, C. J., \& Diamond, P. J. 1998, ApJL, 493, L17

Soifer, B. T., Sanders, D. B., Madore, B. F., et al. 1987, ApJ, 320, 238

Surace, J. A., Sanders, D. B., \& Evans, A. S. 2000, ApJ, 529, 170

Sutherland, R. S., \& Bicknell, G. V. 2007, ApJS, 173, 37

Sutherland, R. S., \& Dopita, M. A. 1993, ApJS, 88, 253

Tremonti, C. A., Moustakas, J., \& Diamond-Stanic, A. M. 2007, ApJL, 663, L77

van Dokkum, P. G. 2001, PASP, 113, 1420

Veilleux, S., Cecil, G., \& Bland-Hawthorn, J. 2005, ARA\&A, 43, 769

Veilleux, S., Cecil, G., Bland-Hawthorn, J., et al. 1994, ApJ, 433, 48

Wilson, C. D., Harris, W. E., Longden, R., \& Scoville, N. Z. 2006, ApJ, 641,763

Wilson, C. D., Rangwala, N., Glenn, J., et al. 2014, ApJL, 789, L36 